\title{
Mobile On-demand Computing: The Future Generation of Cloud
}

\author{
Md. Abdullah-Al-Shafi ${ }^{1 *}$, Ali Newaz Bahar ${ }^{2}$ and Sajeeb Saha ${ }^{3}$ \\ ${ }^{1}$ Institute of Information Technology, University of Dhaka, Dhaka, Bangladesh \\ ${ }^{2}$ Department of Information and Communication Technology, \\ Mawlana Bhashani Science and Technology University, Tangail, Bangladesh \\ ${ }^{3}$ Department of Computer Science and Engineering, Jagannath University, Dhaka, \\ Bangladesh \\ *alshafi08@gmail.com
}

\begin{abstract}
Cloud computing furnishes a unique term of computing and becomes a buzzword to its overwhelming trade prospects. Cloud computing achieved an impetus and is remodeling the internet based computing framework as well as raise the proficiency of mobile systems. Mobile cloud computing or on-demand computing is attaining fame among mobile users and mobile devices can use clouds for various exhaustive applications like storage or information processing. Mobile cloud computing overcomes performancerelated difficulties such as battery and storage capacity as well as communication and environment connected affairs like security, availability, and privacy. Still, mobile ondemand computing beneath anticipation because of privacy and security perils. This paper presents an analysis of mobile cloud computing along with its architectural basis and challenges as well as the security considerations. The objective of this paper is to subsidize a superior perceptive on mobile cloud computing and identify further imminent research directions.
\end{abstract}

Keywords: Cloud computing, Mobile cloud computing, Security affairs, Mobile applications

\section{Introduction}

Cloud computing presents an advanced network application approach and maintains computing infrastructure as well as systems on-demand basis to users. Computing resources like storage and memory are not substantially present at the consumer's locale in on-demand computing, rather the computing sources are owned and operated by service providers such as Amazon EC2 and the users access the systems by the way of the internet. Cloud computing bears along a modern period of improvement of the internet that consents users to employ services on the fly in pay-as-you-go aspect [1] over the internet. The computing paradigm presents abundant favors for businesses, including shorter start-up time for contemporary services and lower process and maintenance cost. Different layered architectures convenient for on-demand computing to serve the aforementioned services as a utility $[2,3]$. Today cloud computing is not only restricted to the particular computer, it also a repercussion and acute impact on the mobile technology such as tablets or smartphones. Mobile cloud computing bear high aspect computational resources to network operators, mobile consumers as well as cloud computing providers by consolidated the mobile computing, cloud computing and wireless networks $[4,5]$. Mobile cloud computing becomes a persuasive movement in modern technology although it faces divers complications in their resources such as battery life and transmissions like security and mobility. The earlier analyses in this field survey this possibility by allowing a mobile device as a consumer and as both consumer and resource provider to cloud platforms. 


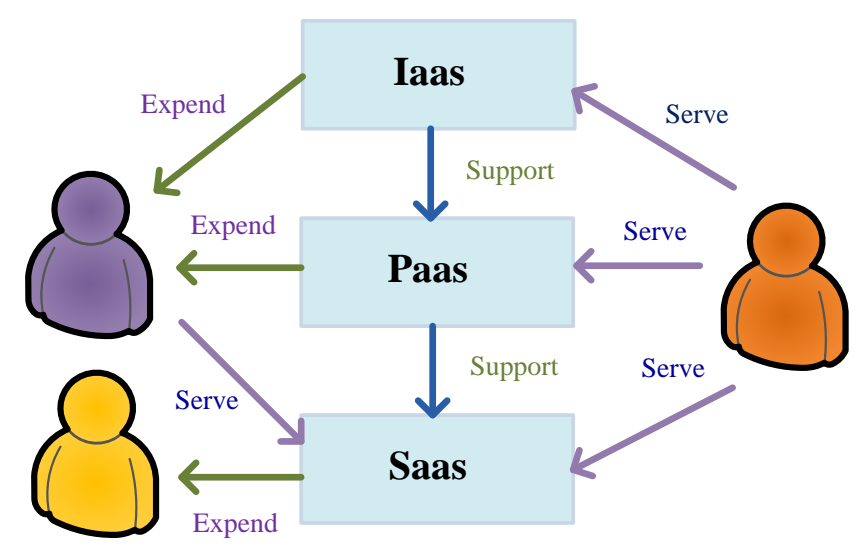

Figure 1. The Structure of Cloud Computing

This paper confers the key architectural model of mobile cloud computing, surveys the system comprehensively as well as the security perspective, and convenience of the mobile cloud is presented. Finally, contemporary research status of mobile cloud computing is conferred.

\section{Mobile Cloud Computing}

The phrase 'mobile cloud computing' was acquainted not long later the idea of 'ondemand computing' which has been draw attention the concentration of producers as a beneficial business opportunity that lessen the advancement and operating expenditure of mobile applications of mobile consumers as a modern technology and analysts as an auspicious elucidation of green IT [6-8]. 'Mobile cloud computing' is a rich computing technology that refers to a framework where both the handling and storage of data take place outside of the device any place, any period through the medium of internet regardless of assorted platforms and environments based on the pay-as-you-use proposition.

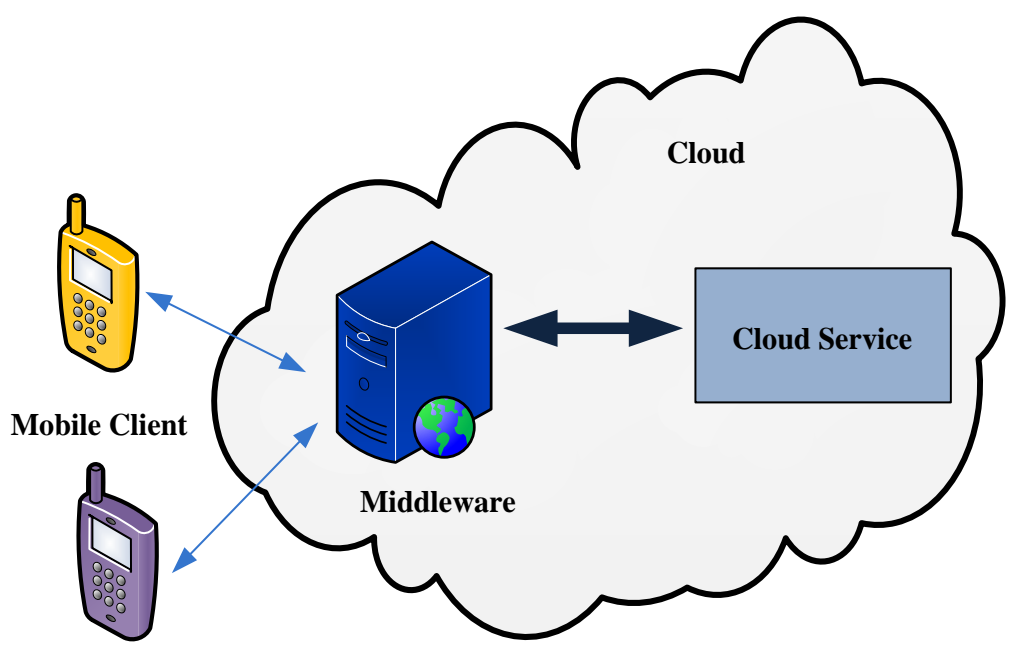

Figure 2. General View of Mobile Cloud Computing 
Mobile on-demand computing can be described as a consolidated of cloud computing and mobile web that is the most prominent device for mobile users to access services and functions on the internet. Other [9] expresses the perception of mobile computing as 'information at fingertips anyplace, anytime'.

\subsection{Architecture of Mobile Cloud Computing}

The architecture of mobile cloud delineates the status of the mobile cloud where client end of a mobile cloud is approximately shifted, but the main concept is still cloud computing. From the idea of mobile cloud, the service proceeding of mobile cloud is that mobile consumers access service directory by interfaces, next the demands of users are sent to the executing system, the system finds out the legitimate data resource by configuration mechanism and uses appropriate system services where the services isolated required resources from the cloud. After web application is beginning, the measuring and monitoring service of the system will pursue the using position of cloud and performs synchronizing composition as well as load balancing composition to make clear factual resource to be allocated to proper consumers. The mobile devices are associated with the mobile networks by way of satellite or access point which control and provide the connections and functional interfaces between the mobile devices and networks.

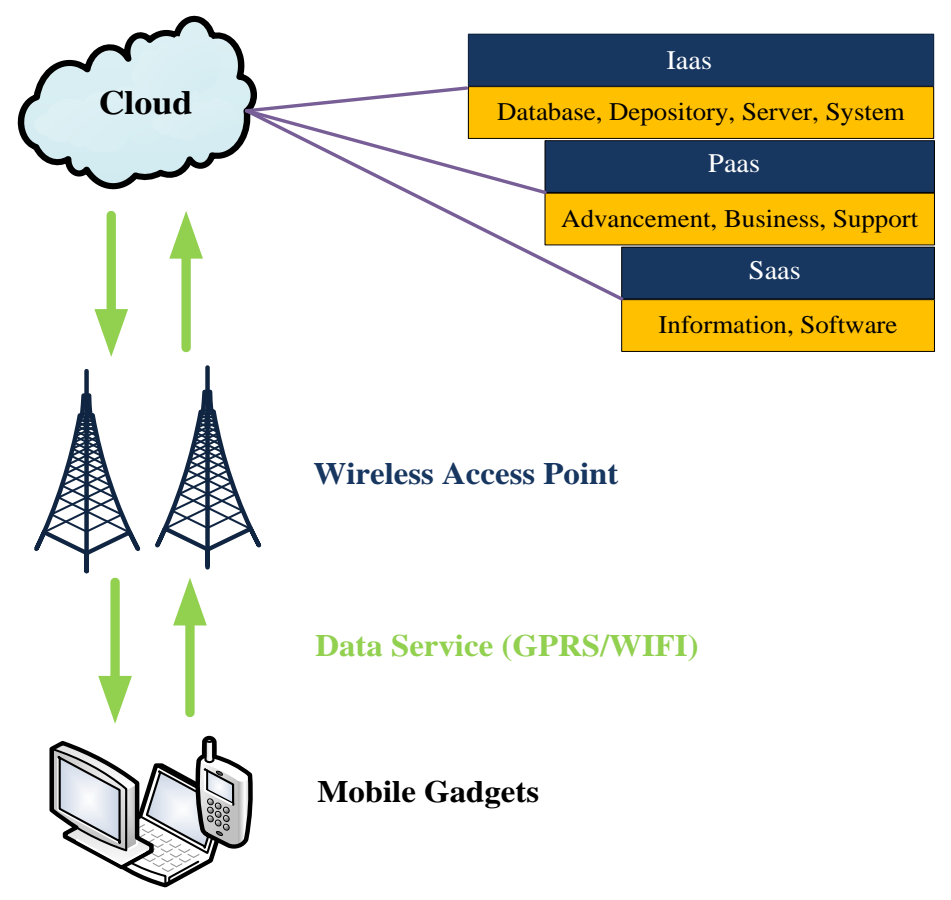

Figure 3. Architecture of Mobile on-Demand computing

Users' requests and data are transferred to the basic processors that are associated with servers presenting network services and network operators can furnish services to mobile consumers as authorization and verification based on the local operator and subscribers' information stored in the directory. Thereafter the subscribers' demands are transferred to a cloud through the internet where the authority proceeding the demands to provide consumers with the reciprocal cloud services. 


\subsection{Characteristics of Mobile Cloud Computing}

The extensive characteristic of mobile cloud computing is listed below:

2.2.1. Competence of Task Handling: The handling capability of cloud structure is pronounced so if the input and output interface is competent, then the consequence of tasks precisely to the cell phones.

2.2.2. Framework Scalability: Recent nodes can be added or released from the network with limited adjustments to the framework set up in physical servers. Mobile cloud structures can comfortably extent vertically or horizontally.

2.2.3. Availability of Sharing Information: An immense volume of data is hoarded in the cloud servers that permits sharing data comfortably. If the frequency range is broad then it will work smoothly, which is simple to comprehend for mobiles.

2.2.4. Wide Network Connection: Consumer competence and proficiency are feasible over the network which can be achieved through assorted platforms like a cell phone or laptop.

2.2.5. Region Independence: Region autonomy is a meaningful distinctive of mobile cloud. There is a sense of diverse position independence where the user typically has no authority over the literal position of the given resources; nevertheless, it can be able to define the area at a higher level of abstraction from state or datacenter.

\subsection{Application: The Obligation for Mobile Cloud}

The uses based on the mobile cloud are ubiquitous. These applications fall into divers' fields like Mobile Learning, Mobile Healthcare, Mobile Game, Mobile commerce besides it influences the area of Social networking, sharing the internet, image processing and so on. An exceptional application of mobile cloud is 'MOTOBLUR' service urged by Motorola.

2.3.1. Mobile Commerce: Mobile commerce or m-commerce concedes business model to use mobile and the applications typically conform few tasks which need mobility like mobile transactions or mobile shopping. An E-commerce platform is proposed [10] where the preference of Cloud Computing and third generation network expand the information processing speed based on public key infrastructure.

2.3.2. Mobile Healthcare: $M$-healthcare diminish the restraints of conventional medical therapy like security and privacy, small-scale physical storage as well as medical omissions [11]. It support users with conducive services to connect resources such as patient health documents or staff profile conveniently.

2.3.3. Mobile Game: Mobile game is also known as $\mathrm{m}$-game which is a promising market producing revenues for the service provider. It entirely offloads game engine needing massive computing resource like graphic rendering to the server in the cloud where gamers simply interact with the screen interface on their gadgets. Multimedia code or offloading [12] can save efficiency for mobile devices, and expanding playing time on mobile devices. Besides, fine-grained energy aware structure is proposed, MAUI [13] which facilitates offloading of mobile codes to a cloud.

2.3.4. Image Processing: An application of image operation is GOCR that is an optical character recognition or OCR program of mobile devices [22]. In a certain life plot, this 
might be helpful in a case of a tourist who takes a photograph of a street sign, executes OCR to excerpt the words, and converts them into a familiar language.

2.3.5. Social Networking: As sharing user content is a prominent way of interacting with associates on social sites such as Facebook or Twitter, incorporating a mobile cloud into social networking platforms might open up automatic sharing access, and this will also dwindle the requirement to back up as well as provide complete information on large servers [10].

\subsection{Operational Issues}

Operational affairs specify to basic technological elements like in what way mobility is maintained, data management and connection pacts operated.

2.4.1. Mobility Management: A crucial affair encountered in a mobile cloud is the layout of the smart mobile management method which provides consumer flexibility while contributing a smooth service. Even though analysis has been consummated in region management in wireless networks [14]. Localization basically categorized either peer-based or framework based techniques where infrastructure-based approaches generally use Wi-Fi, GPS, GSM, RFID ultra-sound with RF procedure. Recent GPS very exceptionally works indoors, and the most precise procedures need supplementary framework as well as need the solid formation of connects points.

2.4.2. Data Management: In many cloud system, maintaining information on the cloud increases many complexities and normally information available to device's holder, would presently accessible to, shared with outside user. Representation of data in a heterogeneous mobile cloud leads to complications with interoperability, flexibility as well as safety and privacy. Computations in a mobile cloud would be extent of a distributed file system and various devices may need to access and customize data.

2.4.3. Network Protocols: Recent mobile cloud analysis operates a variation of network protocols for transmission including Bluetooth, Wi-Fi, and third generation of mobile technology, though the majority has employed Wi-Fi for many reasons.

- Bluetooth: Bluetooth was resolved for nonresident operations and material like wireless headsets and is defined by its low power concerns and low expense transceiver chips [15]. The dimension for Bluetooth is usually in a radius of $10 \mathrm{~m}$ which rely on the interference in the environment, device power, and class. According to Bluetooth specifications, imminent versions will deplete inferior energy and rapid up to $24 \mathrm{Mbps}$.

- Wi-Fi: It admits electronic tools to connect to the network, generally using the 2.4 gigahertz ultra-high frequency and 5 gigahertz super high frequency industrial, scientific and medical (ISM) radio bands. At first, it is resolved for peripheral and cabling resource among computers for wireless local area network or WLAN. Wi-Fi has a high distance, with a radius in $100 \mathrm{~m}$ and supporting up to $11 \mathrm{Mbps}$ data rates.

- Third generation telecommunication: Third generation mobile technology or 3G is a standard technology for mobile service and shares the key business model with that of the telecommunications services model. The framework is maintained and owned by the service provider which is sold out to consumers normally on a monthly usage basis.

A survey of connection protocols with their benefit and loss concerning mobile cloud system is demonstrated in Table 1. 
Table 1. Survey of Connection Protocols used in Various Mobile Clouds

\begin{tabular}{|c|c|c|c|}
\hline Infrastructure & Protocol & Pros & Cons \\
\hline Cuckoo & \multirow{2}{*}{ Bluetooth } & \multirow{2}{*}{$\begin{array}{l}\text { Extensive availability } \\
\text { as opposed to other } \\
\text { protocols, Use low } \\
\text { power }\end{array}$} & \multirow[t]{2}{*}{ Limited extent } \\
\hline MMPI & & & \\
\hline Cuckoo & \multirow{3}{*}{$3 \mathrm{G}$} & \multirow{3}{*}{$\begin{array}{l}\text { Immediate pervasive } \\
\text { coverage }\end{array}$} & \multirow{3}{*}{$\begin{array}{lr}\text { Limited } & \text { bandwidth, } \\
\text { huge } & \text { energy } \\
\text { consumption and low } \\
\text { performance }\end{array}$} \\
\hline Clone Cloud & & & \\
\hline MAUI & & & \\
\hline Chroma & \multirow{6}{*}{ Wi-Fi } & \multirow{6}{*}{$\begin{array}{l}\text { Improved performance } \\
\text { and inferior energy } \\
\text { consumption compared } \\
\text { to } 3 \mathrm{G} \text {, better extent than } \\
\text { Bluetooth }\end{array}$} & \multirow{6}{*}{$\begin{array}{l}\text { Security risk, inter- } \\
\text { operability } \\
\text { between qualities or } \\
\text { deviation may cause } \\
\text { narrow connection or } \\
\text { reduced } \\
\text { momentum }\end{array}$} \\
\hline Cuckoo & & & \\
\hline Hyrax & & & \\
\hline MAUI & & & \\
\hline MobiCloud & & & \\
\hline Scavenger & & & \\
\hline
\end{tabular}

The point of the cellular network like voice telephony or data services has started to draw concentration. Mobile broadband access of various Mbps is feasible via recent $3 \mathrm{G}$ releases often denoted as $3.5 \mathrm{G}$ and $3.75 \mathrm{G}$ [16], even though this is considerably lower than the information rate of $\mathrm{Wi}-\mathrm{Fi}$.

\subsection{Context-aware Service}

Context allowed elements of mobile device permit us to confirm further data from the computing device itself beyond the requirement for accurate user input. The context module can track context criterions and conform to adjustment of context status which facilitated various applications like accurate spatial contexts using Wi-Fi or Bluetooth and Region or Location Based Services (LBS). Context differentiates mobile cloud from typical thoughts.

2.5.1. Common Context Management: Heterogeneous Access Management, an infrastructure [17] for the service of context information presented by the mobile cloud as assistance for a mobile terminal. The urged management architecture is liable for operating, achieving as well as handling and the quality enabler maintains the supplies of context information in accordance with the conditions of the cloud executive. An infrastructure for allowing context-aware mobile business is presented [18] where the infrastructure permits tasks of holding context, resolving what context-particular adaptation is required, and functioning the conformed service.

2.5.2. Social Context Service: Mobile Community Cloud-based Platform [19] may influence the entire potential of mobile community progress besides analyzes the concerns of mobile communities; a cloud model for mobile communities is urged and confers the scientific context of this cloud framework. Smartphones in proximity jointly utilizing their resources to lessen the requirement for the cellular infrastructure to exploit. Here Resource Aware Collaborative Execution is presented which is a Markov decision procedure framework that takes consumers inclination and profile to find out the degree of association. Next, the Resource Aware Collaborative Execution permits the service of mobile devices in the proximity while mobile data transmits. 
2.5.3. Spatial Context Service: A region based data store for cell phones relates with the cloud called 'WhereStore' [20]. The elemental feature of 'WhereStore' is that it uses the cell phone's region history to resolve what information to imitate locally and the objective of caching data on mobile is to decline the general data connect latency as well as lessen the chance of data turn into unavailable in times of no connectivity. A mobile cloud-based collaborative approach for context-aware navigation through exploiting the computation resources and region explicit resources available on the internet. An extensible structure which reduces dependence on infrastructure, so it permits for extensive usability [20].

\section{Issues and Challenges of Mobile Cloud}

In the unification of Mobile cloud and cloud computing, mobile cloud computing front huge scientific affairs and challenges. This section depicts the issue and challenge of mobile cloud communication.

\subsection{Issues in Mobile Cloud System}

Mobile cloud has vast importance for mobile consumers as well as service providers. So in the integration of cloud system, various issues are rising. Some issues in the mobile cloud system are listed here.

3.1.1. Service Availability: Availability becomes a critical affair in the mobile cloud than that in the cloud computing with systems. Consumers may not be capable of associating the cloud to access a service because of network deficiency and congestion of traffic. The resolution aid consumers in the case of cloud disconnection [21] that illustrates a discovery structure to find the nodes in the proximity of a consumer whose channel to the cloud are unavailable. After finding adjacent nodes that are in a fixed form, the target provider for the application is shifted and in this process, a mobile consumer can attach to the cloud through adjoining nodes in an ad hoc aspect. But the ability of device and privacy of adjoining nodes is vulnerable in this structure which is attempted to overcome using a Wi-Fi based network system and a distributed content protocol for the situation beyond any infrastructure. According to the information, every node manages an adjoining node and a content list as well as evaluate role levels of other nodes based on the power supply and bandwidth. Lastly, a node with the highest role level and the abridged hop length are preferred as the transitional nodes to obtain content.

3.1.2. Low Bandwidth: An important affair in the mobile cloud is bandwidth as the radio resource for the wireless system is enough deficient as analyzed with the typical wired networks. An explanation of defined bandwidth is allotment the bandwidth among users of an identical region like a station or organization and engaged in the same content like an audio or video file. A data distribution policy [22] that regulates when and how many parts of accessible bandwidth are shared among consumers from which systems such as Wi-Fi. It gathers user profiles systematically and generates verdict tables by using Markov decision algorithm. Based on the decision, the users resolve whether or not to aid other users to download few contents which they cannot accept by themselves because of the bandwidth restraint.

3.1.3. Offloading Environment: Offloading is one of the key structures of the mobile cloud to increase the battery duration for the mobile devices as well as to improve the attainment of applications. Nevertheless, there are some related affairs including active and proficient offloading beneath environment switches. Offloading can deal with the static or dynamic environment.

Offloading is not consistently the efficient manner to save energy and it might take more efficiency compared to local processing when the content of code is small. For 
instance, offloading expends around 5\% of a mobile battery for transmission, when the altered code content later compilation is $500 \mathrm{~KB}$ and local processing expends around $10 \%$ of the battery for transmission. In this term, offloading can emancipate the battery up to $50 \%$. Gaussian function [23] offloads the total matrix into the distant server. In terms of the energy efficacy, the rate of offloading is higher for trivial matrices, while the rate preserving can be up to $45 \%$ for big matrices. A program splitting [24] based on the estimate of the energy expenditure earlier the program implementation. The optimum program splitting for offloading is computed based on the trade-off between the calculation and transmission rates. Some explanations are urged to find the optimal result for partitioning functions before offloading. A division system to offload computational functions on a mobile device [10] which is based on the profiling data roughly computation period and information sharing at the level of process calls. This process composes a cost graph. Another method to determine [25] which element of Java program would be offloaded and in this approach first the program fragmented into methods which use input criterions to calculate performance costs for these processes. Next, it measures the local performance costs of each system with the distant performance costs which are evaluated based on the position of the recent wireless network. A computation offloading process on a mobile device that urged a polynomial period algorithm to find an optimum program division. This method divided a program into the distributed subprograms where the physical memory reference is mapped into the reference of abstract memory positions. The platform abstraction is developed at a runtime based on pointer survey methods [26].

A scheme to partition an application in dynamic environments where the scheme surveys some phases with various conditions linked to the application configuring and security. In the application configuring phase the programs are organized to be dynamically and effortlessly performed between cloud and mobile device. To attain this, both cloud and consumer essentially have all portions of the application which resolve what units to run the server and client vigorously. In the second step, the structure will select splitting policy so that the entire energy expenditure is lessened and lastly for security concern, modules holding sensitive information will be implemented locally. Though, it lacks efficiency as the partition is based on a prediction model over an off-line scrutiny.

Architecture to vigorously partition an application at runtime covers few stages [21] where the first one generates two kinds of mobile application, one for remote implementation and another for local implementation using code portability. Also, modern smartphones generally use instruction set architecture which is distinct from the server, so MAUI is formed to implement the similar program on distinct architectures. Next, to recognize which approaches of the function are spotted remotely or not as well as type security to excerpt the program state required by the remotely approaches, MAUI operated programing reflection. Finally, the MAUI profiles every mode of an application as well as operates serialization to define communication rates and associates key aspects including energy consumption rate, network status, and communication cost.

3.1.4. Context-Aware Cloud Maintenance: This service accomplishes consumers comfort by controlling their inclinations and supporting proper services to every consumer. In mobile service cloud, protracted from service cloud model a user operates a service on the cloud system and demands to go service gateway. Mobile service cloud forms transitory proxies for devices to check service route as well as maintenance active reconfiguration. A structure for furnishing context-aware service based on the algorithm to select a context-aware connector. Primarily defines a gap following in the specified context where gap stated an outcome of context shifts, next the algorithm defines a reason of predefined gaps earlier preserving the present cases of the service request for improving disconnection. For each of the recognized gap, the algorithm will select a proper adapter for consumers. In the case of consumer inclination, the association might 
be tested when the context of mobile consumer shifts. Though, another context, the association cannot be identified when consumer change to a different context and furthermore the reason, gap, and connector in this model are predefined thus this might lack elasticity in reasonable usage.

\subsection{Challenges in Mobile Cloud System}

3.2.1. Quality of Service: In mobile cloud consumers requires contacting to server positioned in a cloud once requesting resource and service in the cloud system. Yet consumers face few complications like network interruption, and attenuation of the signal caused by consumers' mobility. Latest exploration such as 'Cloudlet' or 'CloneCloud' is projected to diminish network interruption.

CloneCloud transports the power of cloud on smartphones [27]. The concept is to clone the whole set of application and information from the mobile onto the cloud also selectively perform few procedures on the clones, re-assimilating the outcomes back into the mobile.

Cloudlet is a reliable, resource-full computer or group of computers that is extremely associated with the internet and accessible for use by the neighboring mobile device. If no cloudlet is presented adjacent, the device can denote to the default method which will transmit requests to a remote cloud or in the worst case, exclusively its specific resources. A structural design [28] in utilizing virtual machine technology to instantly instantiate modified service software on an adjacent cloudlet and uses that service over a wireless local region network. Though certain attentions which must be focused afore the scheme can be practiced extensively in the functional system like how to distribute storage, processing, and networking ability for each cloudlet?

3.2.2. Motivation for Surrogates: If consumers are to be influenced to share and cooperate their resources with others, there requirements to be incentive either through community or economic motivations to do so. A manner is employing mutual aims [32], however, in the lack of mutual events, this will not prevail. In the case of financial motivations, some problem needs to be response like how will financial connections proceed in a locked process? How will the charge of resources be resolved?

3.2.3. Energy Restraint: Energy in mobile cannot be reinforced instinctively and needs outside resources to be improved. Some efforts have been in advancement to restock efficiency from outside resources such as human motion. Instead, a method like function offloading [28] is projected to preserve mobile resource, particularly energy. But offloading is an uncertain, resource thorough method, which requires improvement and additional research to be employed in actual situations. Scientists $[13,26]$ attempted to alleviate offloading challenge by utilizing flexible, consistent, protected cloud in place of incomplete, uncertain resource but cloud-based function offloading cannot continually hoard energy with recent improvements and requires additional cloud resources instead of insecure, limited surrogates resources.

3.2.4. Cloud Strategy for Mobile Consumers: Cloud suppliers operate several strategies to confine service feature to a preferred level by appointing certain restrictions through their intermediary applications such as Google App Engine bulk loader. Considering the excessive distinctions in wireless and wired transmissions, ignoring resource restrictions and mobility of devices in scheme and support of cloud structures can considerably influence on the feasibility of mobile cloud explanations. Therefore, it is vital to adjust strategies and limitation rules to meet mobile cloud consumers' needs and understand concentrated mobile computing on the go. 


\section{Convenience and Limitation of Mobile Cloud}

Cloud computing reveals an intrinsic conversion in the form information technology and recognized to be an auspicious explanation for mobile cloud owing to several motives. In the following, certain conveniences of the mobile cloud are presented.

- Progressing dependability: Through keeping information on clouds is an efficient practice to enhance the consistency by which the information furthermore application are saved and backed up on a number of computers. Therefore, the risk of information and application missing on mobile devices is lessened and eventually dependability is enlarged.

- Enhancing processing power and data storage facility: Information storage proficiency is a significant constraint for a mobile device. Mobile cloud is improved to increase the mobile consumers to access or hoard vast information on the cloud system through a wireless network. Some instances which are frequently used like Amazon Simple Storage Service to impart file storage on the cloud system.

- Spreading period of battery: Some explanations have been suggested to enhance disk the CPU operation and organizes the disk and screen in a sensible approach to diminish power depletion. To accomplish these explanations might need modifications in the configuration of the mobile device or need an advance tool which results in a progress of charge but these modifications might not be reasonable for all mobile devices. So as to perform the huge calculations and complex handling from a resource-restricted device like mobile devices to ingenious engines like servers in clouds some computations offloading procedure is projected. Mobile cloud eludes taking an extended application implementation period on mobile devices that might effect in a huge quantity of power consumption.

- Effective provisioning: Vigorous provisioning of supplies is a malleable technique for consumers and service providers to run numerous applications without enhanced reservation of resources. Without storing information in a mobile device, it is saved in the cloud and can be approached effectively. The key goal behind the mobile cloud is to support a swift and appropriate technique for the client to accept and access information from the cloud. Largely the challenge of mobile cloud arises from the characters of a mobile device and wireless system as well as their particular limitation and restraint.

The complete limitations of mobile devices, the feature of wireless communication and support from cloud to mobile are all vital issues which concern accessing from cloud computing. Key limitation and explanations of the mobile cloud are listed below.

- Restricted control and elasticity: As each service and applications run on distant or third party virtual locations, consumers have restricted influence over the entire function and implementation of the software and hardware. Furthermore, a distant program is being employed for the mobile cloud, it generally lacks the attributes of an application running regionally.

- Bandwidth hitch: Mobile network is much lesser matched with the conventional networks. Bandwidth is one of the foremost imperative concerns in a mobile cloud. Hence, P2P broadcasting streaming for allocating little bandwidth among the subscriber who is positioned neighboring in the identical region for the similar content like the same video. Employing this technique, every consumer might communicate or exchanges portions of the same content with second consumers, which is appeared in the enhancement of content attribute, particularly for video transmission.

- Dependency and retailer lock-in: The main drawbacks of a mobile cloud is the absolute dependency on the internet provider. It is truly uncomfortable and awkward if 
one consumer wants to shift from one provider to other as he has to transfer a huge amount of information from the former supplier to another one. This is the additional key motive why have to precisely and comprehensively consider all possibilities when selecting a retailer.

\section{Security Issue in Mobile Cloud}

Computing usage has developed a lot started form integrated computing to distributed, internet, mobile extended mobile cloud although safety is still measured to be a serious obstacle for most of the computing systems where the thread and charges have never been entirely removed. Consumers' reliability and secrecy of information or applications are one of the crucial concerns in securing mobile cloud which is the consolidation of mobile network and cloud computing.

\subsection{Mobile Cloud Security}

Mobile cloud safety presents certain encounters [29, 31] that suggest a safety standard for elastic applications composed of 'weblets' which might be transferred to and from a cloud to a mobile.

- Authentication between weblets which would be allocate between the device and the cloud,

- Authentication for weblets that could be performing on comparatively distrusted cloud environments to approach sensitive information and Formation and confirmation of reliable weblet completing cloud nodes.

The safety structure is based on the idea that the cloud pliability service, containing the application manager, cloud manager and cloud fabric interface is reliable. The structure considers several points such as reliable weblet container on cloud and device, locked and confirmation period management required for safe transmission between several instantiations and weblet simultaneously, besides sorting and auditing of weblets. MobiCloud [31] directs to offer a safety facility design for MANET cloud in following techniques.

- Identity controlling is maintained by Attribute based identity management, that provisioned consumer-centric identity patterns also recognized as Identity 2.0. Though the trust authority produces a private key mechanism for every consumer reliant on their public aspect, and the key interchange procedure is not essential.

- MobiCloud has virtual reliable and provisioning domains that are virtual spheres imposed with resource separation. Virtual trusted and provisioning domain encompasses several nodes corresponding to a diverse physical procedure. Nodes in the similarly reliable domain maintenance support the protected transmission when passing information to all other and a cryptography based method is employed to impose information separation and information access control.

- Centralized information accumulation and handling in the MANET is employed by the risk management assistance to recognize pernicious nodes and take deterrent actions as per estimated threats.

A further conceivable explanation is using a method termed 'steganography' and this procedure can be applied to change information like strategies can be accomplished without revealing the information. 


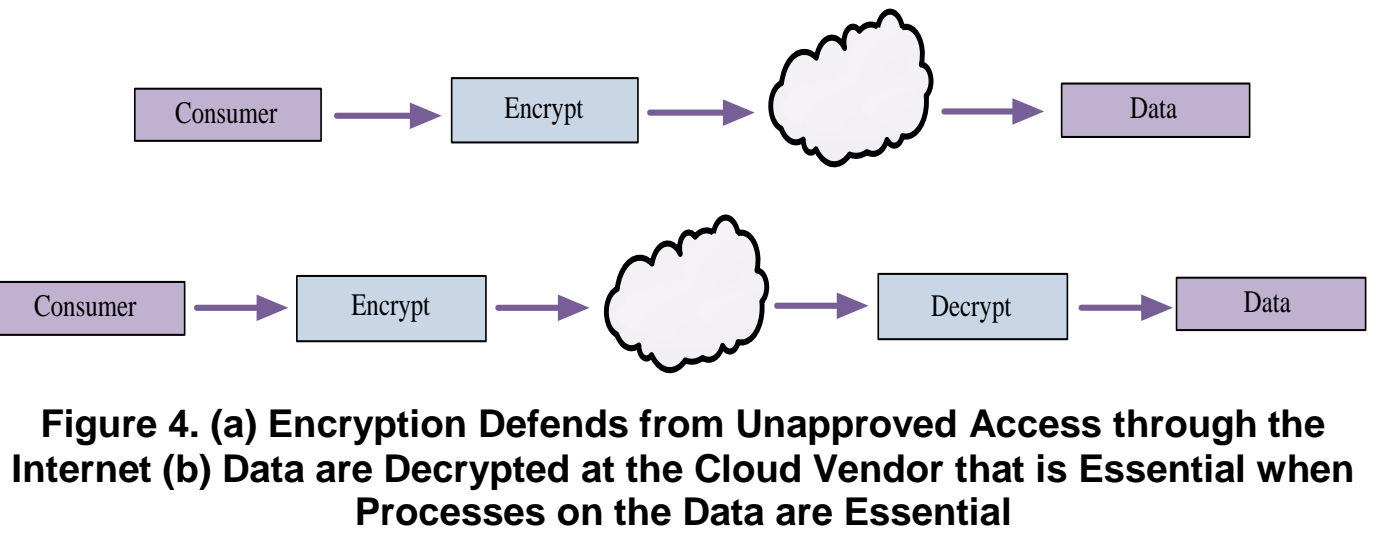

\subsection{Reliability}

Reliability is a potential issue in on-demand computing [1]. A cellphone client executing computation on the cloud is reliant on the cloud service and wireless network. Mobile cloud is strenuous in areas like the underground room or inside of a subway. In this particular circumstance, cloud sometimes unavailable. Reliance on the cloud for essential computations can point to difficulties during the service disruption. Some service contributors like Google have suffered consistency issues. Another difficulty of reliability is data storing which solution is a backup of information with different service contributors.

\subsection{Confidentiality}

Clients' essential information and present position generate circumstances for privacy such as region based services that is offered by global positioning system or GPS. Clients' must be conscious of what particular data is precisely perceptible to the community, and to have managed over their private data that is kept on their devices. Some conditions are presented so far to fulfill clients' confidentiality such as recognition of pirated dataset and defense against wrong use which are effective necessities for mobile cloud too. The pernicious device is a principal affair when sharing data with another device in the mobile cloud and it is the maximum severe matter where the clients' are unspecified and the mobile cloud is designed opportunistically. In this circumstance, the frequency of clients' confirmation requests will rise to such a level that it may perhaps effect in inadequate resources to complete asymmetric key procedures and communicate the compact message. An explanation of this difficulty is PKI based authentication procedure where it offloads the complex PKI processes from the mobile to a remote server. But this process is not feasible for extensive range connectivity. 'Cloudlet' is beneficial for this situation and it could perform as the local infrastructure to which the PKI processes may be offloaded. Unidentified routing is another procedure to provide secrecy for mobile nodes in a distributed mobile cloud such as $\mathrm{p} 2 \mathrm{p}$ domain. Though the certain risk of unpredictable transfer and overheads associated with utmost anonymous $\mathrm{p} 2 \mathrm{p}$ routing procedures and the solution is secrecy must depend on and malleable on the context. For instance, a mobile cloud functioning in community surroundings where the potential for pernicious nodes is extreme must have an extreme point of secrecy, but this would suffer computation rates and upper transmission.

\subsection{Top Threat to Mobile Cloud}

Whether data storage or offloading exhaustive computations through the cloud for a mobile device does pose difficulties of trust affairs and safety. This part describes some maximum threat concerns in mobile computing. 
5.4.1. Information Damage from Stolen or Lost Device: Mobile devices can carry in all places. The data get into through the device denotes that loss or stealing of the mobile device has instant effects. Furthermore, poor key access, and insufficiently or no encryption might precede to data leakage. Clients may also discard or trade devices without understanding the threat to the information. It is a high-level threat and recent risk ensues often so it is the highest affair across administrators and IT organization.

Information loss from decommissioned or stolen devices is a top occurrence affair with both enterprise and employee possessed device. Moreover, traders like Apple have fallen target to stolen or lost patterns of yet to be announced the device.

5.4.2. Data Theft Malware: Numerous download preferences is provided by the android device. Different iOS devices, which must be jailbroken, the android client can simply select to download as well as install application from intermediary marketplace other than 'Play Store' marketplace. The mass of pernicious code allocated for android has been spread through intermediary app stores. Android malware in specific is befitting a more general attack side for offenders who usually have operated a computer as their platforms.

Most frequent pieces of pernicious code for android is 'Zitmo' which is a mobile form of the Zeus malware and devised to filch data from the device by conquering the SMS based banking two-factor permission.

5.4.3. Unsafe Network Access and Wi-Fi: As more clients are mobile and data strategies become more restricted, clients will gradually use Wi-Fi in public places and the result is unsafe Wi-Fi network. This has enlarged the attack surface for clients who link to these networks. Intensified access to free Wi-Fi, along with the enlarged use of mobile devices, generates an increased chance for misuse of this connection.

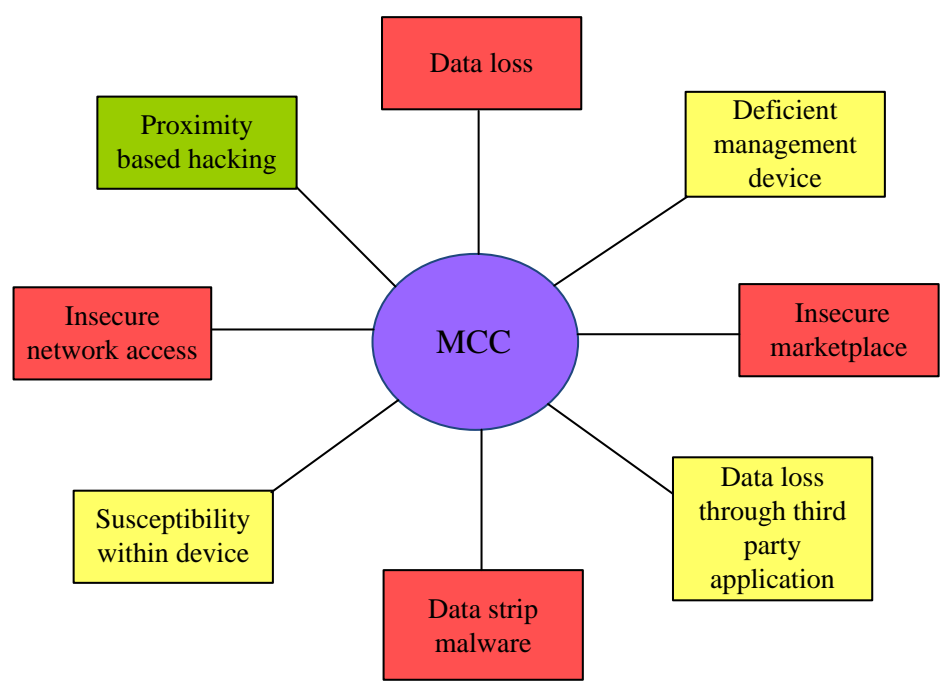

Figure 5. Top Threats in Mobile Computing; Red Box Depicts High-level Risk, Yellow Box Depicts Medium-level Risk and Green Box Depicts Lowlevel Risk

An example is unsafe wireless networks at guesthouses have confirmed to be perfect spaces for hackers to perform an extensive variety of felonies. False Wi-Fi access points are devised to appear real hotel Wi-Fi networks. These malicious systems can cover the hotel's title or other illusive descriptions. 
5.4.4. Rogue Marketplace: All access must me confirmed and authenticated. Smartphone clients, mainly android face difficulties several times to owe to install thirdparty applications. Most of the malware distributed through intermediary stores has been aimed to filch information from the host device. Android malware in specific is being dispensed through this open market more often.

TigerBot' is a bot which is conceived to assemble private information from a device and uses SMS to manipulate the installed bot. This has been exposed on some marketplaces in Asia.

\subsubsection{Information Leakage and Loss through Weakly Written Intermediary Apps:} Tablets and smartphones apps have developed exponentially on android and iOS. Though the main market has safety checks, definite data collection methods are of the uncertain requirement; all too frequently, applications ask for too much approach to data or merely assemble further data than the requirement or else publicize.

In recent times 'LinkedIn' got in some hot water over advantaged access to calendar information within their iPhone and iPad application. Without client realization, LinkedIn's application on iOS device communicated meeting notes, keys, and other data from calendar entries.

5.4.6. Inadequate Supervision Device, Abilities and Access to APIs: Compromising designers and clients contact to a device's low-level functions is a huge complication, as attackers, might also get access to some functions. Moreover, most tablet and smartphone operating systems nowadays, there is slight, if any, guest access or client status. So, all procedure is in the context of the admin, thus offering extreme access in various occurrences.

A client merely leaves the phone unlocked, which permits somebody with access to read as well as change of information on the phone, comprising configuration settings.

5.4.7. Susceptibilities within Devices, Policy, and Intermediary Applications: Third party apps and operating system enfold faults and susceptible to exfiltration of malicious program. The exclusive system innate in mobile offers a particular collection of safety affairs to operating system and application designers as devices progressively cover all of the functionalities accredited to desktop computing with the accumulation of cellular transmission facilities. Illustrations include exponential increase in malware, hardware that guides data back to the constructor, and poor coding practices that are simple to use by felons in third party apps.

5.4.8. Proximity based Hacking and near Field Transmission: Near field communication permits a device to transfer data with another device in small scale wireless technology. It has been utilized in shared media, payment operation, as well as contact information sharing. Because of data worth being communicated, this is expected to be a focus of attackers in the upcoming period. This risk is still in the proof of perception stage.

An effort-by expense arises when, based on the clients' position or vicinity, an attacker may take cash from the clients' digital wallet.

\section{Future Research Issue}

Certain schemes of mobile cloud by now been employed around the world but there is still an extensive path for commercial application and several research attributes should be measured in further effort. The topical issues are still initial periods in mobile computing, including current inductions in the region like CMCVR or MobiSys. This part represents several of the research focuses on the mobile cloud. 


\subsection{Communication Bottleneck Concerns}

Data circulation is extremely climbing by perpetually growing mobile clients' requirements for abusing cloud resources that effect on network operator. Information loading or retrieval, offloading, and live video streaming are sample of mobile cloud processes which radically enhance movement, leads to extreme packet fall and congestion. Therefore, handling such vast data becomes perplexing, particularly when offloading mobile information are dispersed among serving nodes to exchange from the cloud. Though explanations such as employing heterogeneous spectrum and leveraging local hotspots as serving nodes may promote to effortless movement, some outcomes need to be rendered like how protected are the serving nodes and in what extend safety of changed mobile cloud data would be defended? How effective is transmitting offloading data packet? Such types of problems not only need intelligent structures to control offloaded data but also might modify network system and structures general architecture. So, an intellectual system within the mobile cloud that possibly pre-classifies bottleneck concerns and deliberates issues such as mobile cloud application kinds to verify the elite act to relay traffic, is essential as an imminent research objective.

\subsection{Improved Assistance}

The inventive resolution of a mobile cloud is offering Personal computer liked services to mobile stations. Though, as the current changed aspects between the mobile device and Personal computer, cannot exactly relocate the services from Personal computers' platform to mobile device. So, advance research should try to classify the approach on how to offer appropriate and responsive collaborative facilities for the mobile device.

\subsection{Live VM Movement Concerns}

Performing resource concentrated application through VM migration founded application offloading contains the encapsulation of function in VM occasion and roaming it to the cloud that is a perplexing job because of the extra overhead of handling and employing VM on the mobile device [32]. Lengthy expanse raises latency and reduces client perceived application operation which requires migration of VM through geologically dispersed cloud datacenters that are a non-marginal job considering client mobility and wireless communications. Therefore, operative explanations as proactive and responsive VM movement to a position near the client without service disruption becomes crucial to elude client experience deprivation. Attempts like VMware vMotion [33] in the cloud are essential to improve VM migration overhead in the mobile cloud. Decreasing complication and overhead, transmission rate and data volume are acute in short latency, low rate migration of $\mathrm{VM}$ associated with the mobile device.

\subsection{Persuasion and Secrecy Affair}

Confidence is a critical feature for the accomplishment of the flourishing mobile cloud concept. Creating trustable, protected surroundings is an exposed topic that is aggravated when the internet is applied as the connection between front-end and back-end devices. Providing safety, data consistency and integrity besides supplying vital facilities over the heterogeneous systems, wireless networks, and the internet expect original lightweight techniques. Confidence formation based on the provider's status and collection of reliance from each service node would be a precious method that requires forthcoming research. Secrecy is entirely a huge affair in the immense union of some networks, which is aggravated when cloud clients' belief the providers and hoard confidential information on public data warehouses. Therefore, fascinating consumer belief is a significant benchmark 
leading to yet additional challenge, that of how cloud providers can confirm privacy of clients' information.

\section{Conclusion}

Mobile on-demand computing is one of the inclinations in the future since it unites the benefits of both cloud computing and mobile computing, so offering ideal services for mobile consumers. It directs to authorize the mobile client by providing a smooth and ample functionality, regardless of the resource restrictions of mobile devices. Though still in its beginning, the mobile cloud might become the central model for mobile applications in the future. The future might also survey the potential of local mobile clouds designed from groups of computers in pervasive devices in watches, furniture, and other daily items, as certainly, such entrenched computers will become more influential. With the significance, this paper has provided a synopsis of mobile cloud computing in which its definitions, architecture, benefits, challenges, convenience, and security issues have been presented. Lastly, research focuses pertaining to the flourishing area of convergent computing and networking unlocked novel improvement and research opportunities. This work is beneficial for researchers and mobile providers in which they can recuperate their cloud security mechanism and diminish particular client and association mobile cloud computing safety concerns. The forthcoming work will emphasis on how security concern can be upgraded on mobile cloud computing.

\section{References}

[1] M. Armbrust, A. Fox, R. Griffith, A. D. Joseph, R. H. Katz, A. Konwinski, G. Lee, D. A. Patterson, A. Rabkin, I. Stoica and M. Zaharia, "Above the clouds: a Berkeley view of cloud computing", Technical Report UCB/EECS-2009-28, EECS Department, University of California, Berkeley, (2009).

[2] R. Buyya and R. Ranjan, "Federated resource management in grid and cloud computing systems", Future Generation Computer Systems, vol. 26, no. 8, (2006), pp. 1189-1191.

[3] B. Sotomayor, R.S. Montero, I. M. Lorente and I. Foster, "Virtual infrastructure management in private and hybrid clouds", IEEE Internet Computing, vol. 13, no. 5, (2009), pp. 14-22.

[4] S. Abolfazli, Z. Sanaei, E. Ahmed, A. Gani and R. Buyya, "Cloud-based augmentation for mobile devices: motivation, taxonomies, and open challenges", IEEE Communications Surveys \& Tutorials, vol. 16, no. 1, (2014), pp. 337-368.

[5] F. Liu, P. Shu, H. Jin, L. Ding, J. Yu, D. Niu and B. Li, "Gearing resource-poor mobile devices with powerful clouds: architectures, challenges, and applications", IEEE Wireless Communications, vol. 20, no. 3, (2013), pp. 14-22

[6] Shamim, S. M., A. Sarker, A.N. Bahar and M.A. Rahman, "A Review on Mobile Cloud Computing", International Journal of Computer Applications, vol. 113, no. 16, (2015), pp.4-9.

[7] M. Abdullah-Al-Shafi and A. N. Bahar, "Cloud Computing: An Aspect of Information System", International Journal of Applied Information Systems, vol. 10, no. 4, (2016), pp. 46-50.

[8] M. Ali, "Green cloud on the horizon", In IEEE International Conference on Cloud Computing, (2009), pp. 451-459.

[9] M. Satyanarayanan, "Mobile computing: the next decade", ACM SIGMOBILE Mobile Computing and Communications Review, vol. 15, no. 2, (2011), pp. 2-10.

[10] X. Yang, T. Pan and J. Shen, "On 3G mobile e-commerce platform based on cloud computing", 3rd IEEE International Conference on Ubi-media Computing (U-Media), (2010), pp. 198-201.

[11] D. Kopec, M. H. Kabir, D. Reinharth, O. Rothschild and J. A. Castiglione, "Human errors in medical practice: systematic classification and reduction with automated information systems", Journal of Medical Systems, vol. 27, no. 4, (2003), pp. 297-313.

[12] Z. Li, C. Wang and R. Xu, "Computation offloading to save energy on handheld devices: a partition scheme", Proceedings of the 2001 international conference on Compilers, architecture and synthesis for embedded systems (CASES), (2001), pp. 238-246.

[13] E. Cuervo, A. Balasubramanian, C. Dae-ki, A. Wolman, S. Saroiu, R. Chandra and P. Bahl, "MAUI: making smartphones last longer with code offload", Proceedings of the 8th International Conference on Mobile systems, applications and services, (2010), pp. 49-62.

[14] I. Akyildiz, J. McNair, J. Ho, H. Uzunalioglu and W. Wang, "Mobility management in next-generation wireless systems", Proceedings of the IEEE, (1999), pp. 1347-1384

[15] A. Madhavapeddy and A. Tse, "A study of bluetooth propagation using accurate indoor location mapping”, UbiComp 2005: Ubiquitous Computing, Springer Berlin Heidelberg, (2005), pp. 105-122. 
[16] W. Lehr and L.W. McKnight, “Wireless Internet access: 3G vs. WiFi?”, Telecommunications Policy, vol. 27, no. 5, (2003), pp. 351-370.

[17] A. Klein, C. Mannweiler, J. Schneider and H. D. Schotten, "Access Schemes for Mobile Cloud Computing", Eleventh International Conference on Mobile Data Management (MDM), (2010), pp. 387392.

[18] H. J. La and S. D. Kim, “A Conceptual Framework for Provisioning Context-aware Mobile Cloud Services", 3rd International Conference on Cloud Computing (CLOUD), IEEE, (2010).

[19] D. Kovachev, D. Renzel, R. Klamma and Y. Cao, "Mobile Community Cloud Computing: Emerges and Evolves", Eleventh International Conference on Mobile Data Management (MDM), (2010).

[20] P. Stuedi, I. Mohomed and D. Terry, "WhereStore: locationbased data storage for mobile devices interacting with the cloud", Proceedings of the 1st ACM Workshop on Mobile Cloud Computing \& Services: Social Networks and Beyond, (2010).

[21] G. Huerta-Canepa and D. Lee, "A virtual cloud computing provider for mobile devices", Proceedings of the 1st ACM Workshop on Mobile Cloud Computing \& Services: Social Networks and Beyond (MCS), (2010).

[22] E. Jung, Y. Wang, I. Prilepov, F. Maker, X. Liu and V. Akella, "User-profile-driven collaborative bandwidth sharing on mobile phones", Proceedings of the 1st ACM Workshop on Mobile Cloud Computing \& Services: Social Networks and Beyond (MCS), (2010).

[23] A. Rudenko, P. Reiher, G. J. Popek and G. H. Kuenning, "Saving portable computer battery power through remote process execution", Journal of ACM SIGMOBILE on Mobile Computing and Communications Review, vol. 2, no. 1, (1998) pp. 19-26.

[24] K. Kumar and Y. Lu, "Cloud computing for mobile users: can offloading computation save energy", IEEE Computer Society, vol. 43, no. 4, (2010), pp. 51-56.

[25] G. Chen, B. T. Kang, M. Kandermir, N. Vijaykrishnan, M. J. Irwin and R. Chandranouli, "Studying energy tradeoffs in offloading computation/compilation in Javaenabled mobile devices", IEEE Transactions on Parallel and Distributed Systems, vol. 15, no. 9, (2004), pp. 795-809.

[26] R. P. Wilson and M. S. Lam, "Efficient context-sensitive pointer analysis for C programs", Proceedings of the ACM SIGPLAN '95 Conference on Programming Language Design and Implementation (PLDI), (1995), pp. 1-12.

[27] B. G. Chun, S. Ihm, P. Maniatis, M. Naik and A. Patti, "CloneCloud: elastic execution between mobile device and cloud", Proceedings of the 6th conference on Computer systems (EuroSys), (2011), pp. 301314.

[28] M. Satyanarayanan, P. Bahl, R. Caceres and N. Davies, "The case for VM-based cloudlets in mobile computing", IEEE Pervasive Computing, vol. 8, no. 4, (2009), pp. 14-23.

[29] M. Satyanarayanan, "Pervasive computing: vision and challenges", IEEE Pers. Commun, vol. 8, no. 4, (2001), pp. 10-17.

[30] X. Zhang, J. Schiffman, S. Gibbs, A. Kunjithapatham and S. Jeong, "Securing elastic applications on mobile devices for cloud computing", Proceedings of the ACM Workshop on Cloud Computing Security (CCSW), ACM, (2009), pp. 127-134.

[31] A. N. Bahar, M. A. Habib and M. M. Islam, "Security architecture for mobile cloud computing", International Journal of Scientific Knowledge Computing and Information Technology, vol. 3, no. 3, (2013), pp. 11-17.

[32] D. Huang, X. Zhang, M. Kang and J. Luo, "Mobicloud: building secure cloud framework for mobile computing and communication", Proceedings of the 5th IEEE International Symposium on Service Oriented System Engineering, SOSE, (2010), pp. 27-34.

[33] M. Shiraz, S. Abolfazli, Z. Sanaei and A. Gani, "A study on virtual machine deployment for application outsourcing in mobile cloud computing", The Journal of Supercomputing, vol. 63, no. 3, (2013), pp. 946-964.

[34] H. Liu, H. Jin, C.Z. Xu and X. Liao, "Performance and energy modeling for live migration of virtual machines", Proceedings of ACM 20th International Symposium on High Performance Distributed Computing (HPDC), (2011), pp. 171-182.

\section{Authors}

Md. Abdullah-Al-Shafi received his BSc (Engg.) in Information and Communication Technology in 2015. Currently pursuing an MS in Information Technology (IT) in Institute of Information Technology (IIT), University of Dhaka, Bangladesh. He has authored papers in international journals and conference. His research area includes Distributed Computing, Quantum-dot Cellular Automation, Intelligent System and Wireless Sensor Network. https://www.researchgate.net/profile/Abdullah_Al-Shafi 
Ali Newaz Bahar is currently working as assistant professor in the Department of Information and Communication Technology (ICT), Mawlana Bhashani Science and Technology University, Bangladesh. He has more than 30 research publications. His research area includes Quantum-dot Cellular Automation, Big Data Analysis, Distributed Computing and Fuzzy Logic.

https://www.researchgate.net/profile/Ali_Bahar5

Sajeeb Saha is an Assistant Professor in the Department of Computer Science and Engineering at Jagannath University, Dhaka, Bangladesh. Currently he is in study leave to pursue his $\mathrm{PhD}$ degree in the Department of Computer Science and Engineering, University of Dhaka, Bangladesh. His research interests includes Mobile Cloud Computing, Internet of Things, Energy Optimization and Congestion Control Mechanisms. 